\title{
LIGAND-BASED DRUG DESIGN APPROACHES FOR THE IDENTIFICATION OF NOVEL GPR55 MODULATORS \\ GRE ENSBORO
}

LAURA FIGUEROLA-ASENCIO,
$1^{*}$ PAULA MORALES, ${ }^{1}$ DOW P. HURST, ${ }^{3}$ PINGWEI ZHAO, ${ }^{2}$ PATRICIA H. REGGIO, ${ }^{3}$ MARY E. ABOOD, ${ }^{2}$ AND NADINE JAGEROVIC ${ }^{1}$
${ }^{1}$ Instituto de Química Médica, Consejo Superior de Investigaciones Científicas, Madrid, Spain. *I.figuerola@iqm.csic.es
${ }^{2}$ Center for Substance Abuse Research, Temple University, Philadelphia, USA. ${ }^{3}$ Center for Drug Discovery, University North Carolina Greensboro, USA.

\section{INTRODUCTION}

GPR55 is an orphan Class A G-protein coupled receptor that recognizes a sub-set of cannabinoid CB1 and CB2 ligands, suggesting that GPR55 could belong to the endocannabinoid system. Lysophosphatidylinositol (LPI) has been proposed to be endogenous ligand for GPR55. This receptor is involved in diverse physiological and pathological processes such as inflammatory and neuropathic pain, metabolic disorder, bone and neuronal development, and cancer. Few potent GPR55 ligands have been identified to date. High throughput screening of a large library of compounds from the Molecular Libraries Probe Production Centers Network (MLPCN) allowed the identification of different GPR55 Poferer, their p to be optimized in order to develop appropriate pharmacological tools or novel drugs to continue with the challenging goal of the validation of this receptor

\section{AIMS}

In this work, we aim to identify novel potent GPR55 antagonists based on the thienopyrimidine scaffold (hit compound ML192, Fig 1) developing a pharmacophore model that will be used as input for scaffold hopping approaches.

fiig. 1. Structure of the putative endogenous GPR55 ligand LPI, and the GPR55 hit antagonists ML192 identified by a HTS.

\section{$\overline{=}=\overline{=}$ BACKGROUND}

\section{DOCKING AND SAR STUDIES}

|| Previous exploration of the structure-activity relationship (SAR) of the thienopyrimidine scaffold as GPR55 antagonist was focused on modifications at positions $\mathrm{R}^{1}, \mathrm{R}^{2}$ and $\mathrm{R}^{3}$. These modifications were proposed according to the interactions elucidated using docking studies on our GPR55 inactive state model (Figure 2). These compounds were synthesized and evaluated using a $\beta$-arrestin recruitment assay in $\mathrm{CHO}$ cells overexpressing human GPR55 (Table 1).

\section{PHARMACOPHORE MODEL}

The PHASE module integrated in the Maestro software (Schrödinger, LLC, NY 2019) was used to develop of a pharmacophore model (Figure 3) based on the previous SAR data (Table 1).
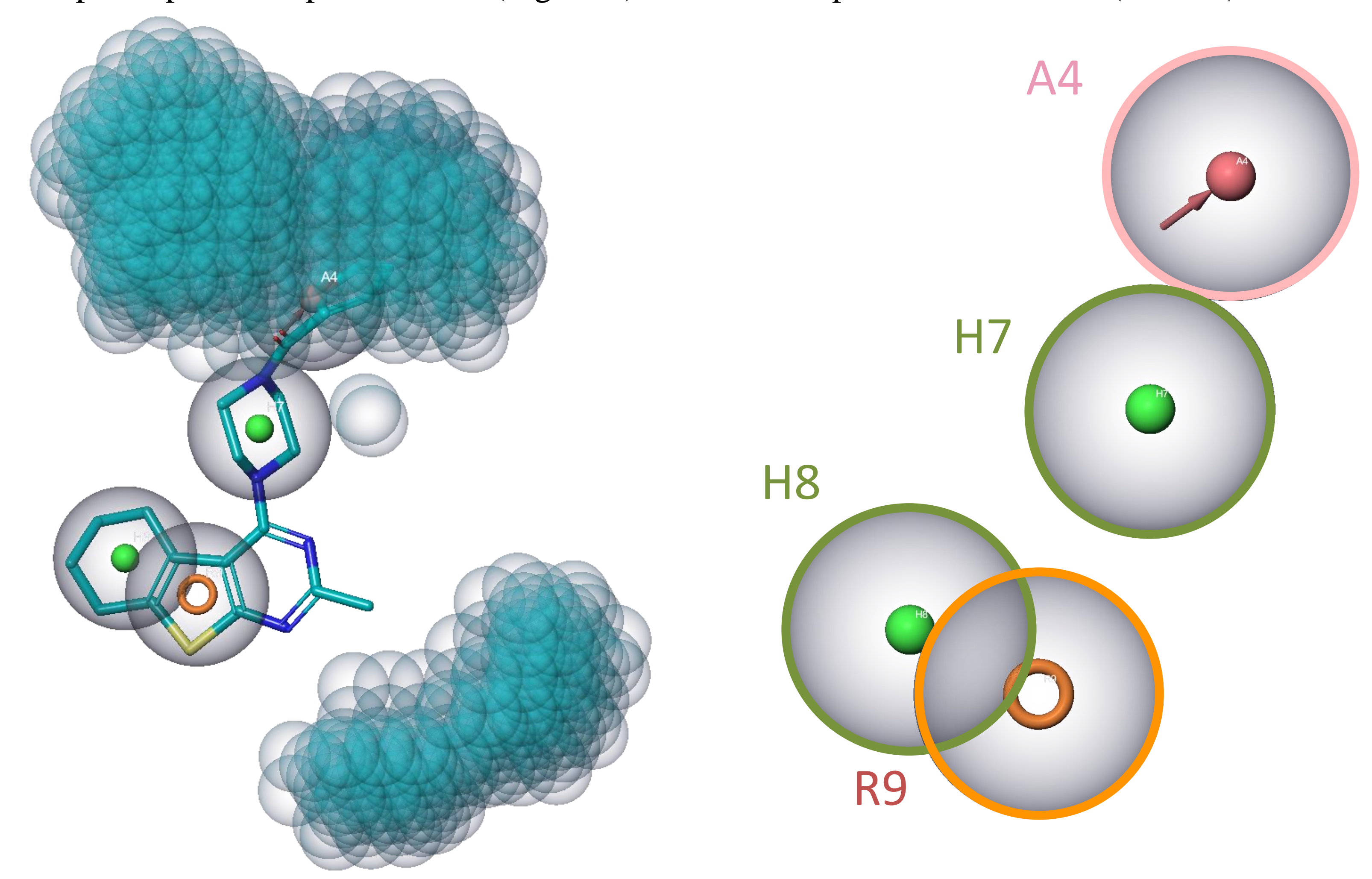

Fig. 3. (A) Visual representation of the thienopyrimidine pharmacophore model. The model contained "excluded

volumes" (Green-blue spheres) simulating the atoms of the binding site surrounding the ligand and, thus, preventing Fig. 2. Docking studies of thienopyrimidine R/ML192 complex; residues interacting with the ligand are shown in blue tubes. (B) $h$ GPR55 R/ML192 complex in which the tubes highlight residues that establish hydrophobic interactions; purple tubes
represent the EC4-EC3 disulfide bridge.

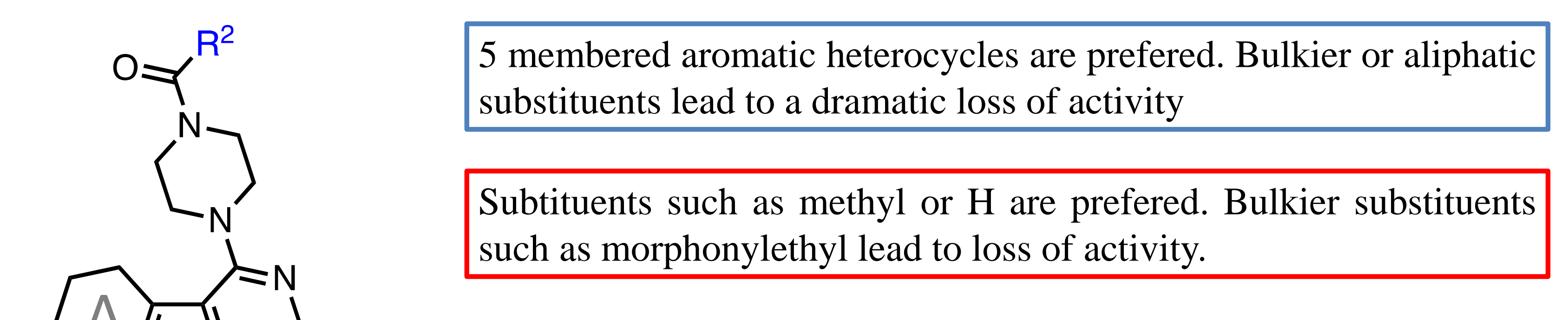

R3 accommodates small substituents but enhanced potency was found for the dimethyl group favoring hydrophobic interactions

\section{PHARMACOLOGICAL EVALUATION}

\begin{tabular}{|c|c|c|c|c|}
\hline Compound & R1 & R2 & R3 & IC50 (mM) \\
\hline ML192 & $\mathrm{Me}$ & Furan-2-yl & $\mathrm{H}$ & $0.70 \pm 0.22 \#$ \\
\hline CID3193014 & Me & Furan-2-yl & $\mathrm{Me}$ & $0.43 \pm 0.11 \#$ \\
\hline CID655864 & Cyclopropyl & Furan-2-yl & $\mathrm{H}$ & $0.86 \pm 0.37 \#$ \\
\hline CID3193022 & Me & Cyclopropyl & Me & $10.6 \pm 2.9 \#$ \\
\hline CID1434957 & $\mathrm{Me}$ & Cyclohexyl & $\mathrm{H}$ & $9.24 \pm 3.76$ \\
\hline CID3193015 & Me & Phenyl & Me & $21.5 \pm 4.6 \#$ \\
\hline CID1434959 & $\mathrm{Me}$ & M-tolyloxymethyl & $\mathrm{H}$ & $>32 \#$ \\
\hline CID3197465 & Me & $\begin{array}{c}\text { 1,2-dimethyl-1H- } \\
\text { benzo[d]imidazol-6-y| }\end{array}$ & $\mathrm{Me}$ & $>32 \#$ \\
\hline CID4877555 & Me & Tetrahydrofuran-2-yl & $\mathrm{H}$ & $>32 \#$ \\
\hline CID46864267 & Me & Pyridin-4-yl & $\mathrm{Me}$ & $>32 \#$ \\
\hline (PM1.4) 15 & $\mathrm{H}$ & Furan-2-yl & $\mathrm{H}$ & $0.68(0.1-3.5)$ \\
\hline (CID1434956) 16 & $\mathrm{Me}$ & Thiophen-2-yl & $\mathrm{H}$ & $3.7(1.8-4.9)$ \\
\hline (LI14) 17 & $\mathrm{Me}$ & 1-methyl-1H-pyrrol-2-yl & $H$ & $13.1(6.3-14.9)$ \\
\hline (KC52) 18 & Me & Furan-2-yl & diMe & $0.28(0.1-0.9)$ \\
\hline (KC59) 19 & Morpholinylmethyl & Furan-2-yl & $\mathrm{H}$ & $2.3(0.1-10.2)$ \\
\hline (SS172) 26* & Me & Furan-2-yl & Me & $8.0(0.7-8.8)$ \\
\hline (SS171) $27^{*}$ & $\mathrm{Me}$ & Furan-2-yl & $\mathrm{H}$ & $3.0(0.1-9.5)$ \\
\hline (LFA258) 34 & 2-hydroxyethyl & Furan-2-yl & diMe & $11.7(3.5-39.1)$ \\
\hline (LFA287B) 35 & Hydroxymethyl & Furan-2-yl & diMe & $7.16(4.5-11.3)$ \\
\hline (LFA273) 36 & Morpholinylethyl & Furan-2-yl & diMe & $>32 \#$ \\
\hline
\end{tabular}

\section{SCAFFOLD HOPPING}

Scaffold Hopping was used to design novel derivatives of the hit compound ML192 based on previous known structural features (Table 1). Isosteric matching core hopping (Maestro software, Schrödinger, LLC, NY 2019) using our developed pharmacophore model was used to explore fragment replacement at specified positions. The piperazine (core A, Fig. 4) and the thienopyrimidine moieties (core B, Fig. 4) were independently studied. Workflow schemes for each core, as well as filtering criteria are detailed in figure 4. Selected final molecules will be synthesized; their structure is not displayed due to potential patentability concerns.

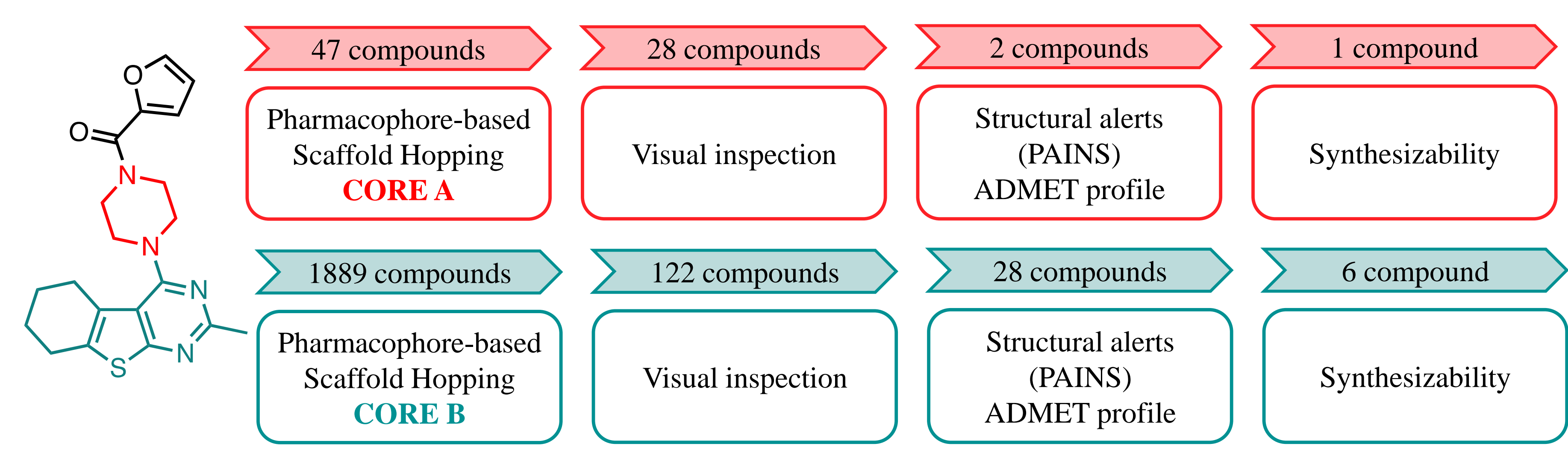

Fig. 4. Workflow scheme of the scaffold hopping process. Filtering criteria
through the isosteric matching core-hopping approach are detailed on each box.

\section{hydrogen bond acceptor; orange, aromatic ring; green, hydrophobic region).}

\section{CONCLUSIONS}

Different computer aided drug design tecniques have been combined to identify novel GPR55 modulators based on the hit antagonist ML192. Derivatization of this compound was approached using isosteric matching core hopping tecniques based on a pharmacophore model developed upon previous SAR studies. Seven different chemotypes have been selected for the initial development following different criteria including structural diversity, ADMET profile and synthetic accessibility. These molecules will be soon synthesized; their structure is not shown due to potential patentability requirements.

References

1. Heynen-Genel, S. et al. (2010) Screening for Selective Ligands for GPR55: Antagonists; NIH; 2. Lingerfelt, M. et al. (2017) Biochem. 56, 473-486; 3. Meza-Avina, E. et al. (2016) Bioorg. Med. Chem. Lett., 26, 1827-1830; 4. Kotsikorou, E. et al. . (2013) Biochem. 52, 9456-9469 